\title{
Activation of transient receptor potential vanilloid 4 induces apoptosis in hippocampus through downregulating PI3K/Akt and upregulating p38 MAPK signaling pathways
}

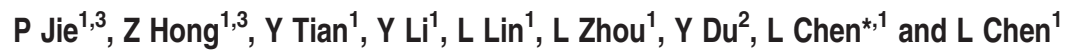

Transient receptor potential vanilloid 4 (TRPV4) is a calcium-permeable cation channel that is sensitive to cell swelling, arachidonic acid and its metabolites, epoxyeicosatrienoic acids, which are associated with cerebral ischemia. The activation of TRPV4 induces cytotoxicity in many types of cells, accompanied by an increase in the intracellular free calcium concentration. TRPV4 activation modulates the mitogen-activated protein kinase (MAPK) and phosphatidyl inositol 3 kinase (PI3K)/ protein kinase B (Akt) signaling pathways that regulate cell death and survival. Herein, we examined TRPV4-induced neuronal apoptosis by intracerebroventricular (ICV) injection of a TRPV4 agonist (GSK1016790A) and assessed its involvement in cerebral ischemic injury. ICV injection of GSK1016790A dose-dependently induced apoptosis in the mouse hippocampi (GSK-injected mice). The protein level of phosphorylated p38 MAPK (p-p38 MAPK) was markedly increased and that of phosphorylated c-Jun N-terminal protein kinase ( $p-J N K$ ) was virtually unchanged. TRPV4 activation also decreased Bcl-2/Bax protein ratio and increased the cleaved caspase-3 protein level, and these effects were blocked by a PI3K agonist and a p38 MAPK antagonist, but were unaffected by a JNK antagonist. ICV injection of the TRPV4 antagonist HC-067047 reduced brain infarction after reperfusion for $48 \mathrm{~h}$ in mice with middle cerebral artery occlusion (MCAO). In addition, HC-067047 treatment attenuated the decrease in the phosphorylated Akt protein level and the increase in p-p38 MAPK protein level at $48 \mathrm{~h}$ after MCAO, while the increase in p-JNK protein level remained unchanged. Finally, the decreased Bcl-2/Bax protein ratio and the increased cleaved caspase-3 protein level at $48 \mathrm{~h}$ after MCAO were markedly attenuated by HC-067047. We conclude that activation of TRPV4 induces apoptosis by downregulating PI3K/Akt and upregulating p38 MAPK signaling pathways, which is involved in cerebral ischemic injury.

Cell Death and Disease (2015) 6, e1775; doi:10.1038/cddis.2015.146; published online 4 June 2015

Transient receptor potential vanilloid 4 (TRPV4), a member of the transient receptor potential (TRP) superfamily, is permeable to calcium $\left(\mathrm{Ca}^{2+}\right) .^{1}$ TRPV4 was first described as a cellular osmotic sensor that detects hypotonic stimulation, and it has now been proven to be activated by multiple stimuli, including mild heat, mechanical stimulation, arachidonic acid (AA) and its metabolites, and exogenous chemical ligands. ${ }^{2}$ TRPV4 is widely expressed in the nervous system and other tissues, including the lungs, bladder and skin. ${ }^{1}$ In the central nervous system, TRPV4 is present in neurons and glial cells. ${ }^{3,4}$ It mediates infrasound- and beta amyloid peptideinduced neuronal impairment, accompanied by an increase in the intracellular free calcium concentration $\left(\left[\mathrm{Ca}^{2+}\right]_{i}\right)^{5,6}$ Application of a TRPV4 agonist dose-dependently induces hippocampal neuronal death in vivo. ${ }^{7}$ Additionally, a gain-offunction mutant of TRPV4 has been shown to augment $\mathrm{Ca}^{2+}$ entry and decrease cell viability in transfected HEK293 cells. ${ }^{8}$ TRPV 4 can be activated by cell swelling-induced mechanical stimulation and metabolites of AA that are always associated with cerebral ischemia. The protein level of TRPV4 has been reported to increase with ongoing reperfusion in a mouse model of middle cerebral artery occlusion (MCAO). ${ }^{7}$ Therefore, the over- or hyper-activation of TRPV4 is likely during cerebral ischemia-reperfusion. Blocking of TRPV4 has been shown to exert neuroprotective effects against cerebral ischemic injury in both in vitro and in vivo studies. . $^{7-11}$ Targeting of TRPV4 is attracting more and more attention in the treatment of cerebral ischemia.

Cell apoptosis, which is one of the major causes of cerebral ischemic injury, becomes prominent after reperfusion for 24-72 $\mathrm{h}^{12}$ It has been reported that excessive $\mathrm{Ca}^{2+}$ entry through TRPV4 leads to apoptosis in mouse retinal ganglion cells, which may be due to the activation of $\mathrm{Ca}^{2+}$-dependent pro-apoptotic signaling pathways. ${ }^{13}$ Mitogen-activated protein kinase (MAPK) signaling pathways that are involved in cerebral ischemic injury have important roles in regulating

\footnotetext{
${ }^{1}$ Department of Physiology, Nanjing Medical University, Nanjing, PR China and ${ }^{2}$ Research Center of lon Channelopathy, Institute of Cardiology, Union Hospital, Tongji Medical College, Huazhong University of Science and Technology, Wuhan, PR China

${ }^{*}$ Corresponding author: L Chen, Department of Physiology, Nanjing Medical University, No. 140, Hanzhong Road, Nanjing, Jiangsu 210029, PR China. Tel/Fax: +86 25 86862878; E-mail:chenl@njmu.edu.cn

${ }^{3}$ These authors contributed equally to this work.

Abbreviations: TRPV4, transient receptor potential vanilloid 4; MAPK, mitogen-activated protein kinase; ICV, intracerebroventricular; JNK, C-Jun N-terminal protein kinase; PI3K, phosphatidyl inositol 3 kinase; Akt, protein kinase B; MCAO, middle cerebral artery occlusion; TRP, transient receptor potential; AA, arachidonic acid;

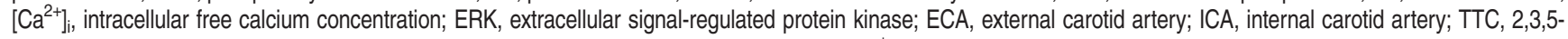
triphenyl-tetrazolium chloride; GAPDH, glyceraldehyde-3-phosphate dehydrogenase; Hoechst ${ }^{+}$, Hoechst-positive

Received 17.2.15; revised 30.3.15; accepted 06.4.15; Edited by A Verkhratsky
} 
cell death and survival through signal translocation pathways related to apoptosis. ${ }^{14}$ The activation of phosphatidyl inositol 3kinase (PI3K)/protein kinase $\mathrm{B}$ (Akt) signaling has been reported to inhibit caspase-dependent apoptosis in cultured neurons and a mouse model of Alzheimer's disease. ${ }^{15-17}$ Activation of TRPV4 can modulate MAPK and PI3K/Akt signaling pathways in different types of cells. ${ }^{7,18}$ In this study, we first assessed the effect of TRPV4 activation on neuronal apoptosis in the hippocampus and then explored the mechanisms underlying TRPV4 action. Finally, we examined the involvement of TRPV4induced apoptosis in MCAO in mice.

\section{Results}

Effect of TRPV4 agonist on apoptosis in hippocampus. In this study, we first examined whether the activation of TRPV4 induces apoptosis in the hippocampus by administering intracerebroventricular (ICV) injections of different doses of the TRPV4 agonist GSK1016790A (GSK-injected mice). The number of Hoechst $^{+}$cells in the hippocampal CA1 area was $4.85 \pm 2.17 / \mathrm{mm}$ in the control group, which is consistent with a previous report. ${ }^{19}$ Figure 1a shows that more Hoechst ${ }^{+}$cells were detected after the mice were injected with GSK1016790A ( $1 \mu \mathrm{M} /$ mouse) compared with the control value. Moreover, at doses ranging from $0.1 \mu \mathrm{M} /$ mouse to $5 \mu \mathrm{M} /$ mouse, GSK1016790A-induced apoptosis was dose dependent, with $\mathrm{EC}_{50}$ values being $1.21 \pm 0.24 \mu \mathrm{M} /$ mouse (Figures $1 \mathrm{~b}$ and $\mathrm{c}$ ). These results provide in vivo evidence that the over-activation of TRPV4 may result in apoptosis in the hippocampus. In the presence of $1 \mu \mathrm{M} /$ mouse GSK1016790A, the number of Hoechst $^{+}$cells in the hippocampal CA1 area was increased by $80.67 \pm 1.16 \%(P<0.01)$, and this dose was used in the following experiments.

Effect of TRPV4 agonist on the expression of apoptosisrelated signaling pathways and apoptosis-related proteins. Among the three MAPK signaling pathways, the p38 MAPK and c-Jun N-terminal protein kinase (JNK) signaling pathways have been implicated in apoptosis in response to stress or some pathological conditions, such as cerebral ischemia. ${ }^{14}$ Modulation of the MAPK signaling pathway by TRPV4 activation has been previously reported. ${ }^{7,18}$ Figures $2 a$ and $b$ show that an increase in phosphorylated p38 MAPK (p-p38 MAPK) protein level was found in the GSK-injected mice, whereas the protein level of phosphorylated JNK1/2 (p-JNK1/2) was nearly unchanged. These results indicate that activation of TRPV4 may enhance the activation of p38 MAPK signaling pathway.

Here, we examined the expression of $\mathrm{Bcl}-2$ and $\mathrm{Bax}$ in the GSK-injected mice. Although the protein levels of $\mathrm{Bcl}-2$ (Figure 2c) and Bax (Figure 2d) were higher in the GSKinjected mice, the $\mathrm{Bcl}-2 / \mathrm{Bax}$ protein ratio was significantly decreased after GSK1016790A treatment $(P<0.01)$ (Figure 2f). In addition, the activation of caspase-3, which is an important mediator of apoptosis, was also assessed. As shown in Figure $2 \mathrm{e}$, a significant increase in the cleaved caspase-3 protein level was found in the GSK-injected mice compared with that in the control mice $(P<0.01)$. In our recent study, activation of TRPV4 has been shown to downregulate PI3K/Akt signaling pathway, which is involved in TRPV4induced neurotoxicity. ${ }^{7}$ Here, it was determined that the decrease in $\mathrm{Bcl}-2 / \mathrm{Bax}$ protein ratio and the increase in the cleaved caspase-3 protein level were markedly rescued in the GSK-injected mice co-injected with 740 Y-P (a PI3K agonist) or SB203580 (a p38 MAPK inhibitor). The change in $\mathrm{Bcl}-2 / \mathrm{Bax}$ protein ratio or in the cleaved caspase-3 protein level in the GSK-injected mice was nearly unaffected by treatment with SP600125 (a JNK inhibitor) (Figures $2 f$ and g). These results imply that activation of TRPV4 may negatively shift $\mathrm{Bcl}-2 / \mathrm{Bax}$ protein ratio and facilitate the activation of caspase-3 protein, which may be related to the inhibited PI3K/Akt and the increased p38 MAPK signaling pathways.
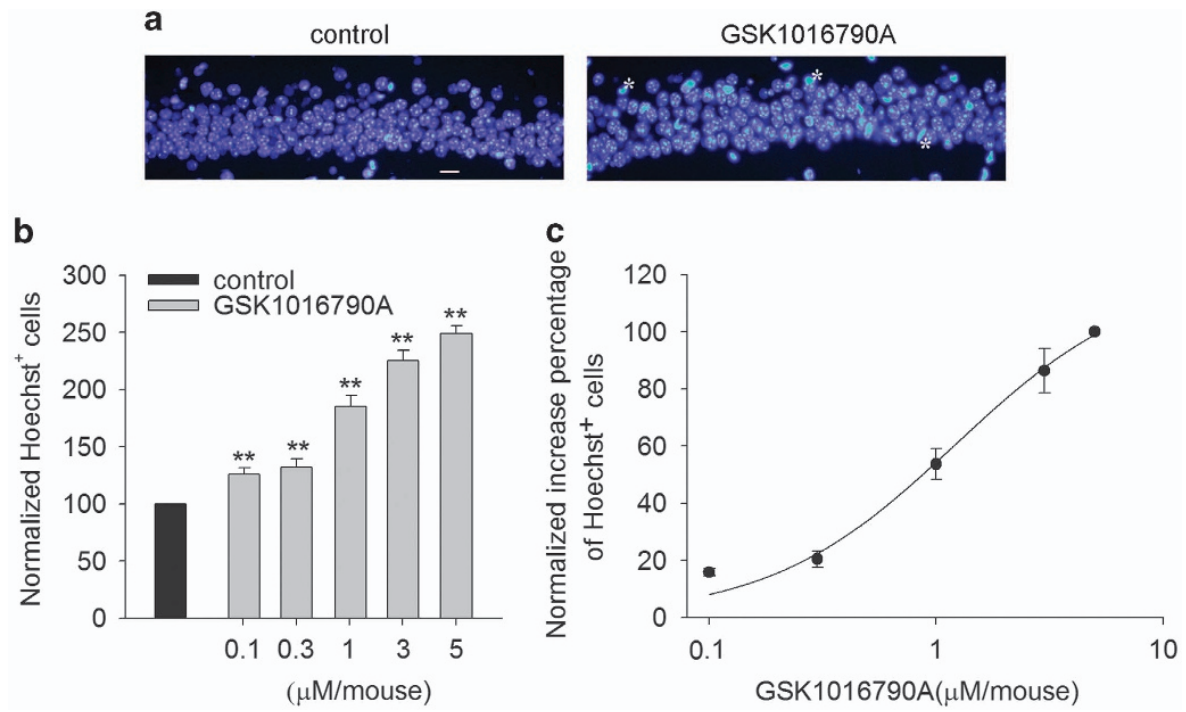

Figure 1 TRPV4-induced apoptosis in hippocampus. (a) Hoechst staining shows that ICV injection of a TRPV4 agonist GSK1016790A (1 $\mu$ M/mouse) induced apoptosis in the hippocampal CA1 area. Scale bar $=50 \mu \mathrm{M}$. (b) The bar graph shows the numbers of Hoechst ${ }^{+}$cells in the hippocampal CA1 area in the presence of different doses of GSK1016790A. ${ }^{* *} P<0.01$ versus control mice. (c) The dose-dependent curve of GSK1016790A-induced apoptosis in the hippocampal CA1 area 
a

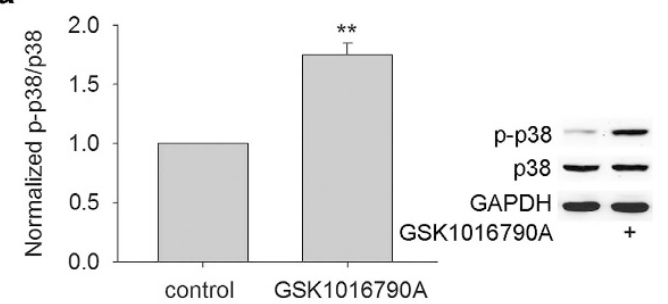

C

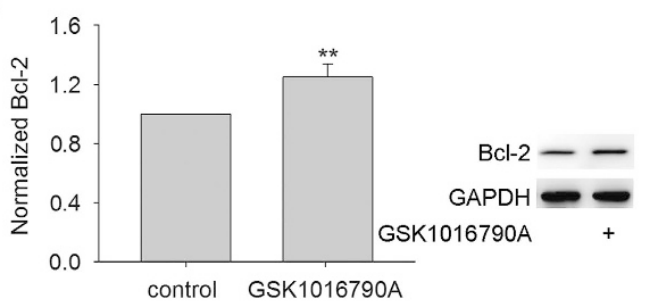

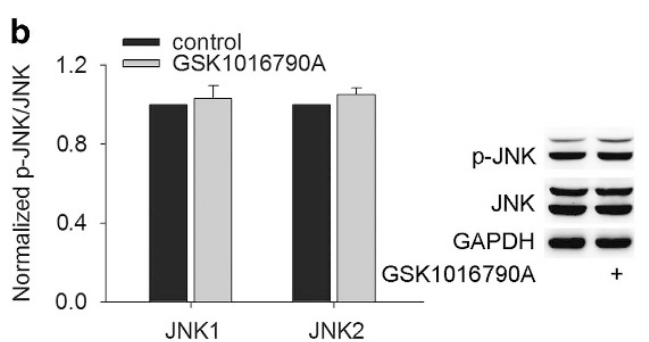

d

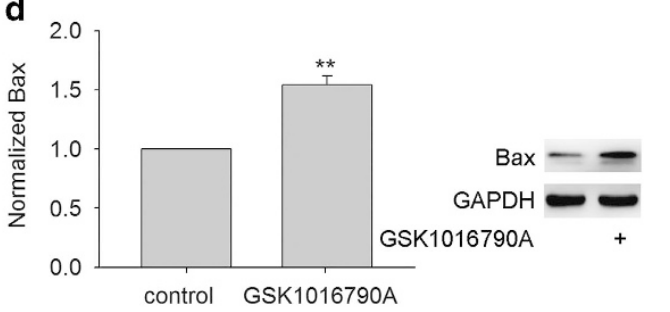

e

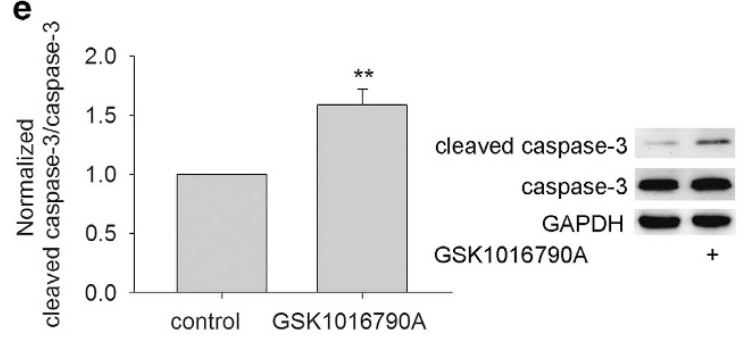

f

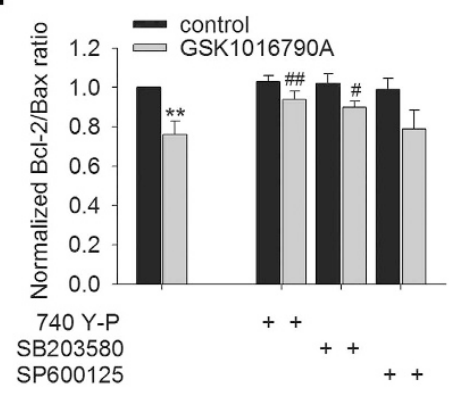

g

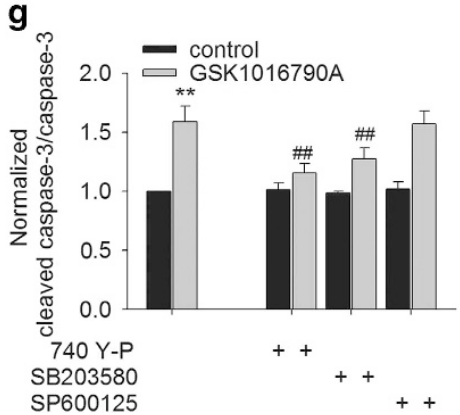

Figure 2 TRPV4-induced modulations of apoptosis-related signaling pathways and apoptosis-related proteins. (a-e) Western blot analysis showing the protein levels of $p$-38 MAPK (a), p-JNK (b), Bcl-2 (c), Bax (d) and the cleaved caspase-3 (e) in the hippocampi of mice injected with vehicle (control mice) and GSK1016790A, respectively. (f and g) TRPV4-induced decrease in Bcl-2/Bax protein ratio (f) and increase in the cleaved caspase-3 protein level (g) were blocked by a PI3K agonist 740 Y-P and a p38 MAPK antagonist SB203580, but were unaffected by a JNK antagonist SP600125. ${ }^{* *} P<0.01$ versus control mice, ${ }^{\#} P<0.05$ and ${ }^{\# \#} P<0.01$ versus vehicle-treated GSK1016790A-injected mice

Involvement of signaling pathways in TRPV4-induced apoptosis in hippocampus. As shown in Figure 3, there were fewer Hoechst $^{+}$cells in the GSK-injected mice co-injected with $740 \mathrm{Y}-\mathrm{P}$ or SB203580 $(P<0.01$ in each case). Additionally, fewer Hoechst ${ }^{+}$cells were found in the mice co-injected with GSK1016790A and Ac-DEVD-CHO (a caspase-3 inhibitor) $(P<0.01)$. By contrast, the number of Hoechst $^{+}$cells in the GSK-injected mice was almost the same as that in the mice co-injected with GSK1016790A and SP600125. Combined with the above results, it is suggested that activation of TRPV4 may downregulate PI3K/Akt and upregulate p38 MAPK signaling pathways to decrease Bcl-2/ Bax protein ratio subsequently and to activate caspase-3 at last, which is likely responsible for TRPV4-induced apoptosis.
Effect of TRPV4 antagonist on brain infarction and changes in the protein levels of p-p38 MAPK, p-Akt and p-JNK at $48 \mathrm{~h}$ post MCAO. Cell apoptosis is evident at 24-72 $\mathrm{h}$ during ischemia-reperfusion. ${ }^{12}$ Here, we examined the effect of a TRPV4 antagonist on brain infarction at $48 \mathrm{~h}$ post MCAO. Figure $4 \mathrm{a}$ shows that the brain infarction volume was $46.67 \pm 3.14 \%$ in the MCAO mice at $48 \mathrm{~h}$ post MCAO. After the MCAO mice were treated with $\mathrm{HC}-067047$, the brain infarction volume was only $12.59 \pm 1.46 \%(P<0.01)$. Here, more Hoechst $^{+}$cells were found at $48 \mathrm{~h}$ post MCAO $(237.01 \pm 15.03 \%)$ and this change was markedly blocked following treatment with HC-067047 (145.11 $\pm 19.21 \%)$ $(P<0.01)$ (Figure 4b). Therefore, it is likely that the apoptosis caused by TRPV4 activation likely contributes, at least in part, to the neuronal injury on the condition of cerebral ischemia. 
We also found that the protein levels of p-p38 MAPK (Figure 5a) and p-JNK (Figure 5b) were markedly increased and that of p-Akt (Figure $5 \mathrm{c}$ ) was decreased significantly at
$48 \mathrm{~h}$ post MCAO. Notably, after the MCAO mice were treated with $\mathrm{HC}-067047$, the increase in p-p38 MAPK protein level and the decrease in p-Akt protein level were obviously rescued, a

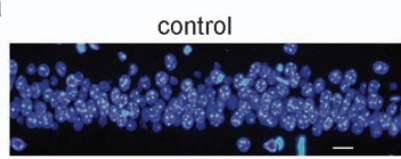

GSK+SB203580

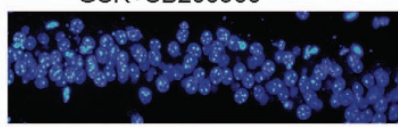

GSK1016790A

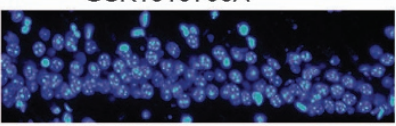

GSK+SP600125

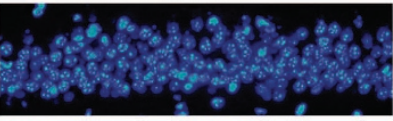

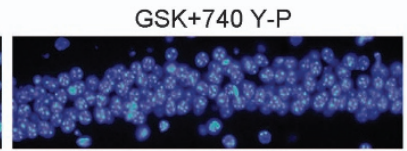

GSK+AC-DEVD-CHO

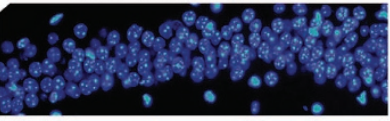

b
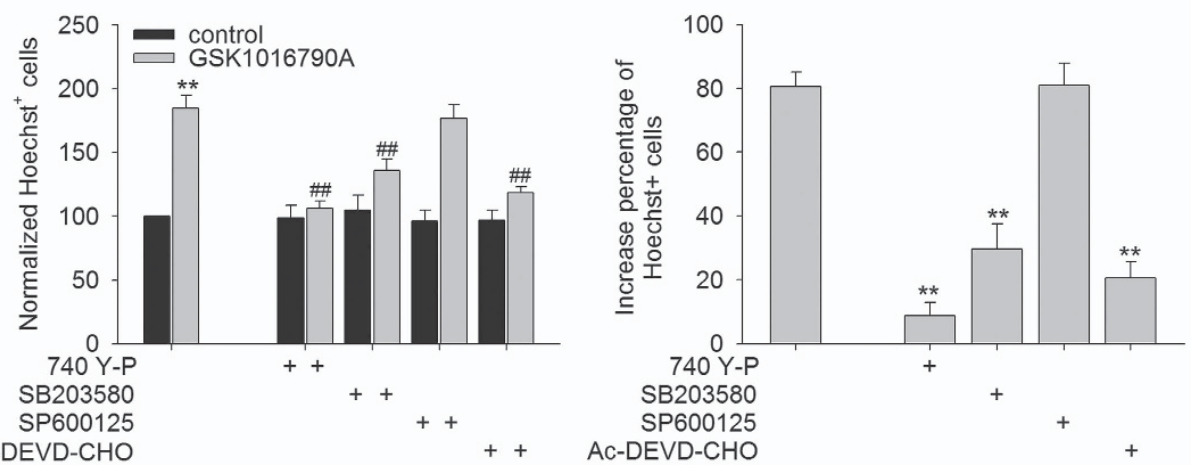

Figure 3 Involvement of signaling pathways in TRPV4-induced hippocampal apoptosis. Hoechst staining (a) and the bar graph (b) show that GSK1016790A-induced apoptosis in the hippocampal CA1 areas was attenuated by 740 Y-P (a PI3K agonist), SB203580 (a p38MPAK antagonist) or Ac-DEVD-CHO (a caspase-3 antagonist), but was unaffected by SP600125 (a JNK antagonist). ${ }^{* *} P<0.01$ versus control mice, ${ }^{\# \# ~} P<0.01$ versus vehicle-treated GSK1016790A-injected mice

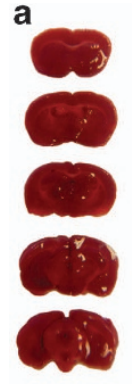

sham-op

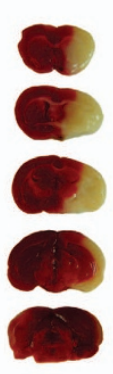

MCAO

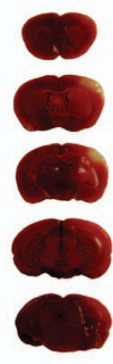

$\mathrm{MCAO} /$ HC-067047
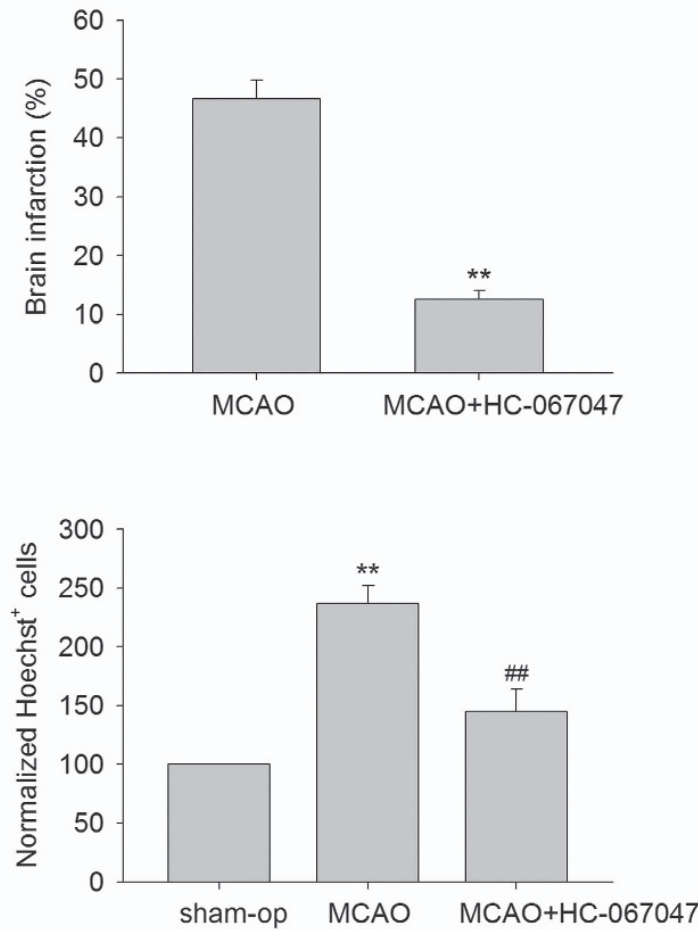

sham-op MCAO MCAO+HC-067047

b

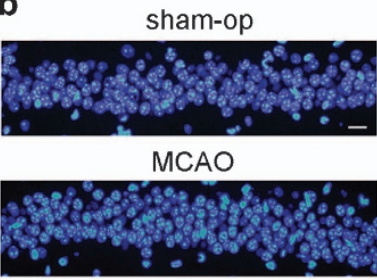

MCAO+HC-067047

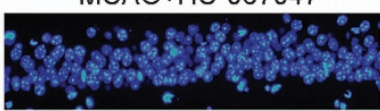

Figure 4 TRPV4 blockage-induced neuroprotection in MCAO mice. (a) Brain infarction at $48 \mathrm{~h}$ post MCAO was reduced by treatment with a TRPV4 antagonist HC-067047. ${ }^{* \star} P<0.01$ versus MCAO. (b) Hoechst staining shows that $\mathrm{HC}-067047$ treatment reduced the number of Hoechst ${ }^{+}$cells at $48 \mathrm{~h}$ post MCAO. Scale bar $=50 \mu \mathrm{M}{ }^{* \star} P<0.01$ versus sham-op, and ${ }^{\# \#} P<0.01$ versus MCAO 


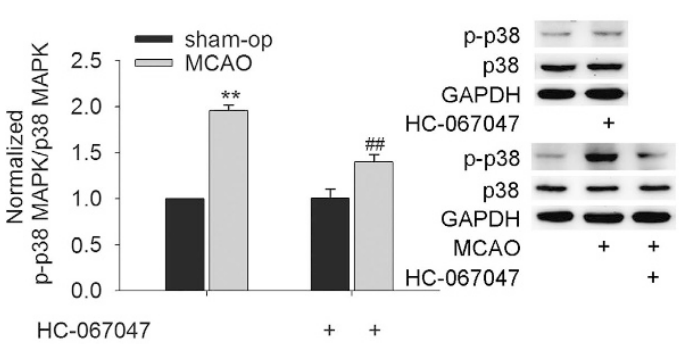

C

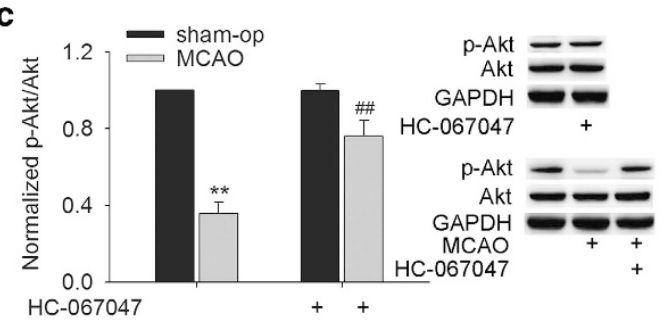

b

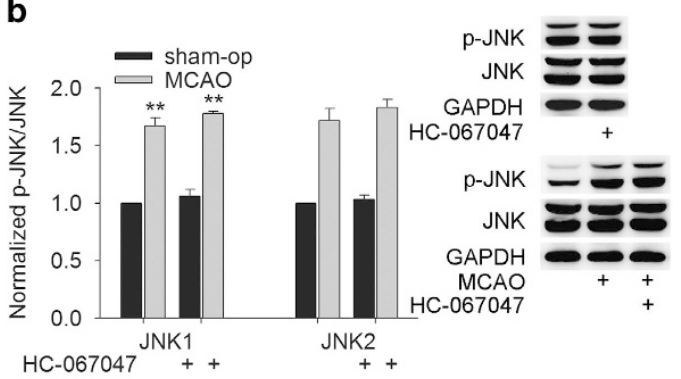

d

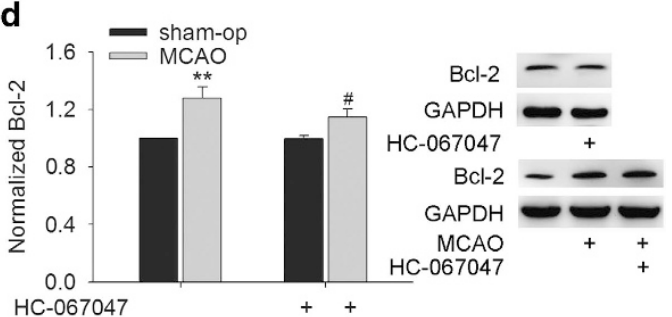

f

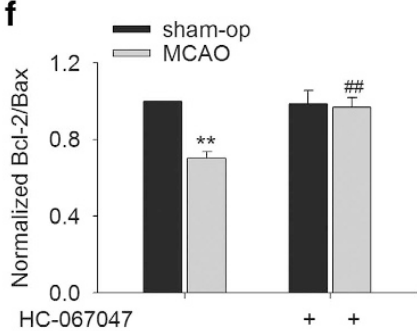

$\mathrm{HC}-067047$ e

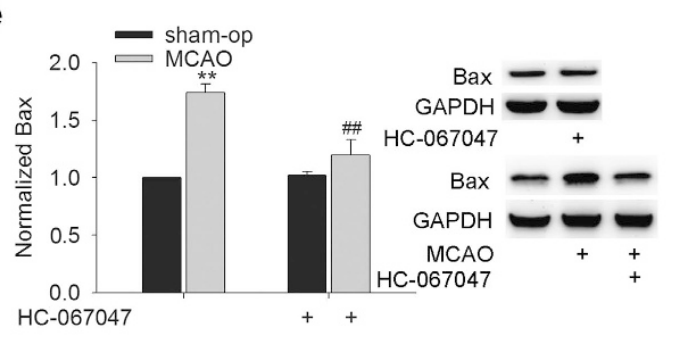

g

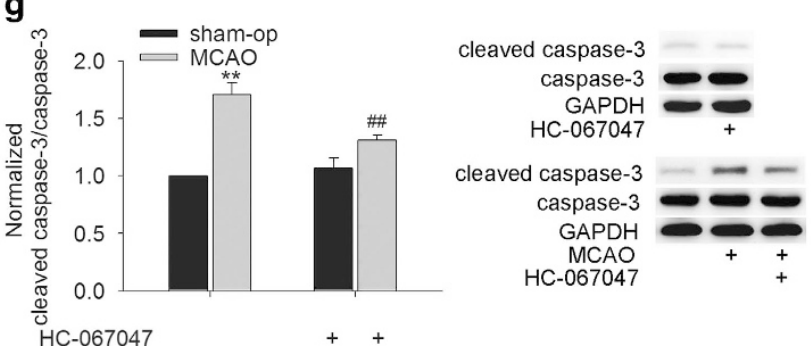

Figure 5 Effect of TRPV4 blockage on apoptosis-related signaling pathways and apoptosis-related proteins in MCAO mice. (a-c) The protein levels of p-p38 MAPK (a) and p-JNK1/2 (b) increased and that of p-Akt (c) decreased at $48 \mathrm{~h}$ post MCAO, but only the increase in p-p38 MAPK and the decrease in p-Akt protein levels were attenuated by HC-067047. (d-f) The increases in Bcl-2 (d) and Bax (e) protein levels at $48 \mathrm{~h}$ post MCAO were blocked by HC-067047. Note that the decrease in Bcl-2/Bax protein ratio (f) in the MCAO mice was markedly attenuated by HC-067047. (g) The increase in the cleaved caspase-3 protein level at $48 \mathrm{~h}$ post MCAO was markedly blocked by HC-067047. ${ }^{* *} P<0.01$ versus sham-op, ${ }^{\#} P<0.05$ and ${ }^{\# \#} P<0.01$ versus MCAO

whereas the increase in p-JNK protein level was nearly unaffected. Additionally, the decrease in Bcl-2/Bax protein ratio and the increase in the cleaved caspase-3 protein level in the MCAO mice were markedly attenuated by HC-067047 treatment (Figures $5 \mathrm{~d}$ and $\mathrm{g}$ ). These results indicate that during the cerebral ischemia, the activation of TRPV4 probably downregulates PI3K/Akt and upregulates p38 MAPK signaling pathways to decrease the $\mathrm{Bcl}-2 / \mathrm{Bax}$ protein ratio and activate caspase-3.

\section{Discussion}

TRPV4 is sensitive to various types of stimuli, including hypoosmotic stimulation, mechanical force, the metabolism of
AA and synthetic ligands. It is a calcium-permeable channel, and a TRPV4-induced increase in $\left[\mathrm{Ca}^{2+}\right]_{i}$ has been confirmed by many research groups. ${ }^{1,2}$ Increasing evidence of TRPV4 activation-related cellular toxicity is emerging. For example, sustained exposure to TRPV4 agonists has been shown to evoke the dose-dependent apoptosis of retinal ganglion cells, accompanied by an elevation in $\left[\mathrm{Ca}^{2+}\right]_{\mathrm{i} \cdot}{ }^{13}$ TRPV4 has been demonstrated to be involved in human islet amyloid polypeptide-triggered apoptosis in a mouse pancreatic beta cell line and to be responsible for infrasound-induced apoptosis in the hippocampus. ${ }^{5,20}$ A TRPV4 mutation displaying an increase in calcium channel activity results in increased cytotoxicity. ${ }^{8,21}$ Consistent with our recent report concerning 
TRPV4-induced neuronal injury, ${ }^{7}$ the present data showed that application of a TRPV4 agonist dose-dependently induced apoptosis in the hippocampus (Figure 1), further demonstrating the neuronal cytotoxicity caused by TRPV4 activation.

Apoptosis is a type of programmed cell death, which is an initiative suicide process that occurs after cells receive a signal or stimulation. MAPKs are a family of serine/threonine protein kinases that are critical for the transduction of signals from the cell surface to the nucleus. Among the members of the MAPK family, extracellular signal-regulated protein kinase (ERK) is mainly responsible for the control of growth and differentiation, and the other two MAPK family members, JNK and p38, have roles in apoptosis as well as in inflammation, growth and differentiation. ${ }^{22}$ Akt has an important role in the suppression of apoptosis. After Akt is phosphorylated by PI3K, it can inhibit cell death by inactivating apoptogenic factors. ${ }^{23}$ It has been reported that Akt signaling pathway may be inhibited by the treatment with a TRPV4 agonist, and ERK signaling pathway may be activated by TRPV4 activation. ${ }^{7,18}$ However, blockage of ERK pathway fails to attenuate TRPV4-induced hippocampal neuronal death. ${ }^{7}$ Therefore, the present study focused on exploring the involvement of Akt, JNK and p38 MAPK pathways in TRPV4-induced apoptosis. First, our data found that the protein level of p-p38 MAPK increased markedly, whereas that of $p$-JNK remained nearly unchanged in the GSK-injected mice (Figures $2 a$ and $b$ ), indicating that activation of TRPV4 selectively activates p38 MAPK signaling pathway. Second, in the presence of a PI3K agonist (740 Y-P) or a p38 MAPK antagonist (SB203580), GSK1016790Ainduced apoptosis was significantly attenuated. By contrast, the apoptosis caused by TRPV4 activation was unaffected by a JNK antagonist (SP600125) (Figure 3). These results indicate that the inhibition of PI3K/Akt and the increase in p38 MAPK signaling pathways are responsible for TRPV4 activation-induced apoptosis.

$\mathrm{Bcl}-2$ family proteins are key regulators of apoptosis and include both anti-apoptotic members, such as Bcl-2, and proapoptotic members, such as Bax. ${ }^{23,24}$ Here, the protein level of Bax was increased to a greater extent compared with that of $\mathrm{Bcl}-2$ in the GSK-injected mice, leading to a decrease in Bcl-2/ Bax protein ratio (Figures $2 \mathrm{~d}$ and f). Caspases are a family of intracellular proteins that are involved in the initiation and execution of cell apoptosis. Caspase- 3 is a potent, terminal caspase that executes apoptosis via a mitochondrialdependent pathway. ${ }^{25}$ The present study showed an increase in the cleaved caspase-3 protein level in the GSK-injected mice, and fewer Hoechst ${ }^{+}$cells were found in mice co-injected with GSK1016790A and caspase-3 inhibitor (Ac-DEVD$\mathrm{CHO}$ ), indicating that TRPV4-induced apoptosis is caspase3 dependent (Figures $2 e$ and 3 ). The above results indicate that activation of TRPV4 may result in a negative shift in the dynamic balance of the Bcl-2 family and ultimately activate caspase- 3 that executes the apoptosis. Here, the decrease in $\mathrm{Bcl}-2 / \mathrm{Bax}$ protein ratio and the increase in the cleaved casapse-3 protein level in the GSK-injected mice were markedly attenuated by a PI3K agonist and a p38 MAPK antagonist (Figure 2g). Collectively, our data suggest that the over-activation of TRPV4 may inhibit PI3K/Akt and enhance p38 MAPK signaling pathways to negatively shift Bcl-2/Bax protein ratio and to ultimately activate caspase-3, which is probably involved in TRPV4-induced apoptosis.

There is increasing evidence that TRPV4 is a promising target for the treatment of cerebral ischemia. First, pathological changes that occur during ischemia/reperfusion, including cytotoxic cell swelling and disturbances in membrane lipid metabolism may facilitate the activation of TRPV4. Second, the protein level of TRPV4 increases with ongoing ischemiareperfusion. Third, blocking TRPV4 has been proven to have protective effects on neurons following oxygen-glucose deprivation treatment or in rodent models of acute cerebral ischemia. ${ }^{6,7,9-11}$ Cell apoptosis is one of the major causes of cerebral ischemic injury, and it may be mediated through MAPK signaling pathways. ${ }^{14}$ The present study showed that application of a TRPV4 antagonist reduced brain infarction and apoptosis at $48 \mathrm{~h}$ post MCAO (Figure 4), indicating that TRPV4-induced apoptosis is likely involved in the cerebral ischemic injury. Here, the increase in p-p38 MAPK and the decrease in $\mathrm{p}$-Akt protein levels in the MCAO mice were markedly rescued by HC-067047. Additionally, the decrease in $\mathrm{Bcl}-2 / \mathrm{Bax}$ protein ratio and the increase in the cleaved caspase-3 protein level in the MCAO mice were significantly inhibited following HC-067047 treatment (Figure 5). In summary, it is proposed that during the cerebral ischemia, the overactivation of TRPV4 results in caspase-3-dependent apoptosis through inhibiting PI3K/Akt and enhancing p38 MAPK signaling pathways, and this action contributes to cerebral ischemic neuronal injury.

The present study was a subsequent work of our previous report in which activation of TRPV4 shows neurotoxicity through enhancing the NR2B subunit of N-methyl-D-aspartate receptor and the related downregulation of Akt signaling pathway and thus is involved in the cerebral ischemic injury. ${ }^{7}$ In the previous study, we have proven that Akt but not ERK signaling pathway is selectively responsible for TRPV4induced neuronal injury, providing a basis for further exploring the role of Akt signaling in TRPV4-induced apoptosis. In this study, p38 MAPK signaling was also confirmed to be responsible for TRPV4-induced apoptosis, whereas the involvement of JNK signaling was excluded. Furthermore, the inhibition of PI3K/Akt and the increase in p38 MAPK signalings were implicated in TRPV4-modulated apoptosisrelated protein expression, including $\mathrm{Bcl}-2$, Bax and cleaved caspase-3. In our previous study, brain infarction at $24 \mathrm{~h}$ post MCAO is reduced by TRPV4 antagonist HC-067047. Cell apoptosis becomes prominent after reperfusion for $24-72 \mathrm{~h}$ following cerebral ischemia; ${ }^{12}$ therefore, we examined the effect of HC-067047 on brain infarction and apoptosis at $48 \mathrm{~h}$ post MCAO in this study. Different time point post-MCAO chosen to study the effect of HC-067047 in cerebral ischemic injury was due to the different research objective in our previous study and the present one.

TRPV4 is widely expressed in the nervous system. Given its sensitivity to diverse stimuli and permeability to calcium, TRPV4 may have an important role in modulating the function of the nervous system under physiological and pathological conditions. In addition to its involvement in cerebral ischemic injury, the potential role of TRPV4-induced neurotoxicity has been reported in $A D$ and motor neuron disease. ${ }^{6,8,26} \mathrm{An}$ increase in TRPV4 expression has been found in the brains of 
aged rats. ${ }^{3}$ Therefore, targeting of TRPV4 provides a promising neuroprotective treatment.

\section{Materials and Methods}

Animals. Male mice (ICR, Oriental Bio Service Inc., Nanjing, China) weighing 25-30 g were used in this study. The animals were housed under a 12:12-h light dark cycle and were provided food and water ad libitum. All animal experiments were approved by the animal experimental committee of Nanjing Medical University, China. Each experimental group contained nine mice.

Drug treatment. All drugs were intracerebroventricularly injected as previously reported. ${ }^{7}$ After the mice were anesthetized with $2 \%$ chloral hydrate $(20 \mathrm{ml} / \mathrm{kg})$, they were placed in a stereotaxic device (Kopf Instruments, Tujunga, CA, USA). A 23-G stainless-steel guide cannula (Plastics One, Roanoke, VA, USA) was inserted into the right lateral ventricle $(0.3 \mathrm{~mm}$ posterior, $1.0 \mathrm{~mm}$ lateral, and $2.5 \mathrm{~mm}$ ventral to the bregma) and anchored to the skull with four stainless steel screws and dental cement. Drugs were injected using a 26-G stainless-steel needle (Plastics One). The drugs were first dissolved in DMSO and then in $0.9 \%$ saline to a final volume of $2 \mu$ with a final DMSO concentration being 1\%. SB203580 (1 mM/mouse), SP600125 (10 $\mu \mathrm{g} /$ mouse), 740 Y-P (30 $\mu \mathrm{M} /$ mouse) or Ac-DEVD-CHO ( $10 \mu \mathrm{M} /$ mouse) was injected 30 min before GSK1016790A injection and subsequently injected once daily for 3 days. The doses of the above antagonists or agonists were chosen as previously described. ${ }^{10,27-30}$ The TRPV4 antagonist HC-067047 was used as previously reported, except that it was repeatedly injected until $48 \mathrm{~h}$ post MCAO. ${ }^{10}$

Preparation of the focal cerebral ischemia model. Transient focal cerebral ischemia was induced by MCAO as previously described. ${ }^{7}$ Briefly, after the mice were anesthetized, the right common carotid artery, external carotid artery (ECA) and internal carotid artery (ICA) were separated and exposed. The origin of the middle cerebral artery was occluded by a poly--Llysine-coated nylon monofilament thread, which was inserted through the ECA and advanced into the ICA. Reperfusion was established by withdrawing the thread after $60 \mathrm{~min}$ of occlusion. The adequacy of vascular occlusion and reperfusion in the front parietal cortex of the occluded side was monitored using a multichannel laser Doppler flowmeter (Perimed PF5050, Jarfalla, Sweden). Body and head temperatures were thermostatically controlled and arterial blood pressure and gases were monitored through a femoral catheter during the operation. Mice in the sham-operated (sham-op) group were treated identically except for the occlusion of the middle cerebral artery.

Infarction volume measurement. Brain infarction was determined using 2,3,5-triphenyl-tetrazolium chloride (TTC) staining as previously described. ${ }^{10}$ The brains were removed at $48 \mathrm{~h}$ post MCAO, sectioned into 2-mm-thick coronal slices and then incubated with a $2 \%$ TTC solution for $20 \mathrm{~min}$. Brain infarction was visualized with image analysis software (MCID; Imaging Research, Canada), and infarct volume was determined as a percentage of the infarct area relative to the contralateral hemisphere area for each slice.

Western blot analysis. Western blot analysis was performed at $48 \mathrm{~h}$ post MCAO or on day 3 after GSK1016790A injection. Hippocampal protein concentrations were determined with a BCA Protein Assay Kit (Pierce, Rochford, IL, USA). Equal amounts of protein were separated by SDS-polyacrylamide gel electrophoresis and transferred onto PVDF membranes. The membranes were blocked using nonfat milk in Tris-buffered saline (TBS)/Tween-20 and then incubated with antibodies against phospho-p38 MAPK (p-p38 MAPK, 1:1000, Cell Signaling Technology, Beverly, MA, USA), p38 MAPK (1:1000, Cell Signaling Technology, Boston, MA, USA), phospho-JNK1/2 (p-JNK1/2, 1:1000, Cell Signaling Technology, Boston, MA, USA), JNK1/2 (1:1000, Cell Signaling Technology, Boston, MA, USA), cleaved caspase-3 (1:1000, Cell Signaling Technology, Danvers, MA, USA), caspase-3 (1:1000, Cell Signaling Technology, Danvers, MA, USA), Bax (1:1000, Cell Signaling Technology, Boston, MA, USA), Bcl-2 (1:1000, Cell Signaling Technology, Boston, MA, USA) and glyceraldehyde-3-phosphate dehydrogenase (GAPDH) (1:5000, Abcam, Cambridge, MA, USA) at $4^{\circ} \mathrm{C}$ overnight. After being washed with TBST, the membranes were incubated with an HRP-labeled secondary antibody, developed using an ECL Detection Kit (Amersham Biosciences, Piscataway, NJ, USA) and analyzed using Image $\mathrm{J}$ software $(\mathrm{NIH})$. Hippocampal samples collected from the hemispheres of three mice were considered a set for western blot analysis. The summarized data represent the average of three experimental sets.
Hoechst staining. On day 3 after GSK1016790A injection or at $48 \mathrm{~h}$ post $\mathrm{MCAO}$, mice were anesthetized and perfused with ice-cold phosphate-buffered saline (PBS) followed by $4 \%$ paraformaldehyde. The brains were removed and immersed in fixative $\left(4^{\circ} \mathrm{C}\right.$ overnight) and then processed for paraffin-embedding. Coronal sections $(5 \mu \mathrm{m})$ cut from the level of the hippocampus were stained with Hoechst-33342. Hoechst-positive (Hoechst ${ }^{+}$) cells were counted using a fluoresce microscope (Olympus PD70) ( $\times 40$ objective). Hoechst ${ }^{+}$cells were counted in six sections per mouse and expressed as the number of cells per millimeter of length along the hippocampal CA1 pyramidal layer. ${ }^{19}$

Data analysis. Data are expressed as means \pm S.E.M. and were analyzed with Stata 7.0 software (STATA Corporation, College Station, TX, USA). ANOVA followed by Bonferroni's post hoc test was used for statistical analysis, and significance levels were set at $P<0.05$ and $P<0.01$. The number of the Hoechst ${ }^{+}$cells or the protein level in the mice that were injected with GSK1016790A or/and kinase agonist/ antagonist was expressed as a percentage of that in the vehicle-injected mice (control mice). The number of the Hoechst ${ }^{+}$cells or the protein level in the MCAO mice or the MCAO mice treated with HC-067047 was expressed as a percentage of that in the sham-op mice. The increases in the number of Hoechst ${ }^{+}$cells resulting from different doses of GSK1016790A were first normalized to the increase caused by $5 \mu \mathrm{M}$ GSK1016790A. The dose-response curve was then fitted by the Hill equation, in which $a=a_{\max } /\left[1+\left(\mathrm{EC}_{50} / C\right)^{n}\right]$, with $n$ being the Hill coefficient, and $\mathrm{EC}_{50}$ being the dose of GSK1016790A producing 50\% effect.

\section{Conflict of Interest}

The authors declare no conflict of interest.

Acknowledgements. This work was supported by National Natural Science Foundation of China (31271206), Qing Lan Project of Jiangsu province (2014-2017) and Research Award Fund for Outstanding Young Teachers in Nanjing Medical University (JX2161015033) to Lei Chen, and National Natural Science Foundation of China (81470421) to Yimei Du.

1. Garcia-Elias A, Mrkonjić S, Jung C, Pardo-Pastor C, Vicente R, Valverde MA. The TRPV4 channel. Handb Exp Pharmacol 2014; 222: 293-319.

2. Vincent F, Duncton MA. TRPV4 agonists and antagonists. Curr Top Med Chem 2011; 11: 2216-2226.

3. Lee JC, Choe SY. Age-related changes in the distribution of transient receptor potential vanilloid 4 channel (TRPV4) in the central nervous system of rats. J Mol Histol 2014; 45: 497-505.

4. Shibasaki K, Ikenaka K, Tamalu F, Tominaga M, Ishizaki Y. A novel subtype of astrocytes expressing TRPV4 (transient receptor potential vanilloid 4) regulates neuronal excitability via release of gliotransmitters. J Biol Chem 2014; 289: 14470-14480.

5. Shi M, Du F, Liu Y, Li L, Cai J, Zhang GF et al. Glial cell-expressed mechanosensitive channel TRPV4 mediates infrasound-induced neuronal impairment. Acta Neuropathol 2013; 126: $725-739$

6. Bai JZ, Lipski J. Involvement of TRPV4 channels in A $\beta(40)$-induced hippocampal cell death and astrocytic $\mathrm{Ca}(2+)$ signaling. Neurotoxicology 2014; 41: 64-72.

7. Jie P, Lu Z, Hong Z, Li L, Zhou L, Li Y et al. Activation of transient receptor potential vanilloid 4 is involved in neuronal injury in middle cerebral artery occlusion in mice. Mol Neurobiol 2014; e-pub head of print 18 November 2014; doi:10.1007/s12035-014-8992-2.

8. Klein CJ, Shi Y, Fecto F, Donaghy M, Nicholson G, McEntagart ME et al. TRPV4 mutations and cytotoxic hypercalcemia in axonal Charcot-Marie-Tooth neuropathies. Neurology 2011; 76: 887-894

9. Lipski J, Park TI, Li D, Lee SC, Trevarton AJ, Chung KK et al. Involvement of TRP-like channels in the acute ischemic response of hippocampal CA1 neurons in brain slices. Brain Res 2006; 1077: 187-199.

10. Li L, Qu W, Zhou L, Lu Z, Jie P, Chen L et al. Activation of transient receptor potential vanilloid 4 increases NMDA-activated current in hippocampal pyramidal neurons. Front Cell Neurosci 2013; 7: 17-26.

11. Butenko O, Dzamba D, Benesova J, Honsa P, Benfenati V, Rusnakova V et al. The increased activity of TRPV4 channel in the astrocytes of the adult rat hippocampus after cerebral hypoxia/ischemia. PLoS One 2012; 7: e39959.

12. Dirnagl U, ladecola C, Moskowitz MA. Pathobiology of ischaemic stroke: an integrated view. Trends Neurosci 1999; 22: 391-397.

13. Ryskamp DA, Witkovsky P, Barabas P, Huang W, Koehler C, Akimov NP et al. The polymodal ion channel transient receptor potential vanilloid 4 modulates calcium influx, spiking rate and apoptosis of mouse retinal ganglion cells. J Neurosci 2011; 31: 7089-7101.

14. Nozaki K, Nishimura M, Hashimoto N. Mitogen-activated protein kinases and cerebral ischemia. Mol Neurobiol 2001; 23: 1-19. 
15. Zhang L, Qu Y, Tang J, Chen D, Fu X, Mao M et al. PI3K/Akt signaling pathway is required for neuroprotection of thalidomide on hypoxic-ischemic cortical neurons in vitro. Brain Res 2010; 1357: 157-165.

16. Yu W, Mechawar N, Krantic S, Quirion R. $\alpha 7$ Nicotinic receptor activation reduces $\beta$-amyloidinduced apoptosis by inhibiting caspase-independent death through phosphatidylinositol 3-kinase signaling. J Neurochem 2011; 119: 848-858.

17. Yang $R$, Chen $L$, Wang $H, X u B$, Tomimoto $H$, Chen L. Anti-amnesic effect of neurosteroid PREGS in A $25-35$-injected mice through $\sigma 1$ receptor- and $\alpha 7 n A C h R-m e d i a t e d$ neuroprotection. Neuropharmacology 2012; 63: 1042-1050.

18. Ye L, Kleiner S, Wu J, Sah R, Gupta RK, Banks AS et al. TRPV4 is a regulator of adipose oxidative metabolism, inflammation and energy homeostasis. Cell 2012; 151: 96-110.

19. Li L, Xu B, Zhu Y, Chen L, Sokabe M, Chen L. DHEA prevents A 25 -35-impaired survival of newborn neurons in the dentate gyrus through a modulation of PI3K-Akt-mTOR signaling. Neuropharmacology 2010; 59: 323-333.

20. Casas S, Novials A, Reimann F, Gomis R, Gribble FM. Calcium elevation in mouse pancreatic beta cells evoked by extracellular human islet amyloid polypeptide involves activation of the mechanosensitive ion channel TRPV4. Diabetologia 2008; 51: 2252-2262.

21. Fecto F, Shi Y, Huda R, Martina M, Siddique T, Deng HX. Mutant TRPV4-mediated toxicity is linked to increased constitutive function in axonal neuropathies. J Biol Chem 2011; 286: 17281-17291.

22. Harper SJ, Wilkie N. MAPKs: new targets for neurodegeneration. Expert Opin Ther Targets 2003; 7: 187-200.

23. Noshita N, Lewén A, Sugawara T, Chan PH. Evidence of phosphorylation of Akt and neuronal survival after transient focal cerebral ischemia in mice. $J$ Cereb Blood Flow Metab 2001; 21: 1442-1450.

24. Ola MS, Nawaz M, Ahsan $\mathrm{H}$. Role of $\mathrm{Bcl}-2$ family proteins and caspases in the regulation of apoptosis. Mol Cell Biochem 2011; 351: 41-58.

25. Wang X. The expanding role of mitochondria in apoptosis. Genes Dev 2001; 15: 2922-2933.
26. Lee JC, Joo KM, Choe SY, Cha Cl. Region-specific changes in the immunoreactivity of TRPV4 expression in the central nervous system of SOD1(G93A) transgenic mice as an in vivo model of amyotrophic lateral sclerosis. J Mol Histol 2012; 43: 625-631.

27. Zhao L, Liu X, Liang J, Han S, Wang Y, Yin Y et al. Phosphorylation of p38 MAPK mediates hypoxic preconditioning-induced neuroprotection against cerebral ischemic injury via mitochondria translocation of Bcl-xl in mice. Brain Res 2013; 1503: 78-88.

28. Sherrin T, Blank T, Hippel C, Rayner M, Davis RJ, Todorovic C. Hippocampal C-Jun-Nterminal kinases serve as negative regulators of associative learning. J Neurosci 2010; 30 13348-13361.

29. Rosenblat M, Volkova N, Paland N, Aviram M. Triglyceride accumulation in macrophages upregulates paraoxonase 2 (PON2) expression via ROS-mediated JNK/c-Jun signaling pathway activation. Biofactors 2012; 38: 458-469.

30. Yin X, Zhang J, Li X, Liu D, Feng C, Liang R et al. DADS suppresses human esophageal xenograft tumors through RAF/MEK/ERK and mitochondria-dependent pathways. Int $\mathrm{J}$ Mol Sci 2014; 15: 12422-12441.

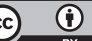

Cell Death and Disease is an open-access journal published by Nature Publishing Group. This work is licensed under a Creative Commons Attribution 4.0 International License. The images or other third party material in this article are included in the article's Creative Commons license, unless indicated otherwise in the credit line; if the material is not included under the Creative Commons license, users will need to obtain permission from the license holder to reproduce the material. To view a copy of this license, visit http://creativecommons.org/licenses/by/4.0/ 